\title{
An eigenproblem approach to classical screw theory
}

\author{
Sandipan Bandyopadhyay* \\ Ashitava Ghosal \\ Department of Engineering Design Department of Mechanical Engineering \\ Indian Institute Technology- Madras \\ Indian Institute of Science \\ Chennai 600036, India \\ Bangalore 560012, India
}

\begin{abstract}
This paper presents a novel algebraic formulation of the central problem of screw theory, namely the determination of the principal screws of a given system. Using the algebra of dual numbers, it shows that the principal screws can be determined via the solution of a generalised eigenproblem of two real, symmetric matrices. This approach allows the study of the principal screws of the general two-, three-systems associated with a manipulator of arbitrary geometry in terms of closed-form expressions of its architecture and configuration parameters. We also present novel methods for the determination of the principal screws for four-, five-systems which do not require the explicit computation of the reciprocal systems. Principal screws of the systems of different orders are identified from one uniform criterion, namely that the pitches of the principal screws are the extreme values of the pitch.

The classical results of screw theory, namely the equations for the cylindroid and the pitch-hyperboloid associated with the two- and three-systems respectively have been derived within the proposed framework. Algebraic conditions have been derived for some of the special screw systems. The formulation is also illustrated with several examples including two spatial manipulators of serial and parallel architecture respectively.
\end{abstract}

\footnotetext{
${ }^{*}$ Corresponding author, email: sandipan@iitm.ac.in.
} 


\section{Introduction}

The theory of screws has been used to analyse finite and instantaneous motions of rigid bodies over the past few centuries. One of the major applications of screw theory has been in describing the instantaneous motion of a rigid body in terms of the principal screws, which form a canonical basis of the motion space. The elements of screw theory emerged from the works of Giulio Mozzi [1] (1763 A.D.), Cauchy, and Chasles [2] (1830 A.D.). However, in 1900, Sir Robert Ball [3] established formally the theory of screws and applied it to the analysis of rigid-body motions of multiple degrees-of-freedom. The fundamental concepts of classical screw theory, including the principal screws of a screw system, the cylindroid, the pitch hyperboloid, and the reciprocity of screws were introduced in his treatise. In 1976, Hunt [4] rejuvenated screw theory from geometric considerations and applied it to the analysis and synthesis of mechanisms.

The determination of the principal screws of a given system has attracted a significant amount of research ever since Hunt's contribution. It is well-known that for the general twodegrees-of-freedom rigid-body motion, the instantaneous screw axis (ISA) lies on a ruled surface (known as the cylindroid) generated by the two principal screws of the system. For general three-degrees-of-freedom rigid-body motion, the three principal screws meet orthogonally at a point. The points on the resultant ISA having the same pitch lie on a quadratic surface, known as the pitch hyperboloid. Hunt [4], Hunt and Gibson [5] have also discussed the special cases of various screw systems and proposed a classification of various screw systems.

Interestingly, in spite of the long history of classical screw theory and the number of contributions in the area of principal screws, there seems to be a lack of variety in the reported works. Researchers have mostly retraced the geometric construction of the principal screws in one way or the other. For example, Zhang and $\mathrm{Xu}[6]$ have reconstructed the pitch hyperboloid algebraically for a general three-system from the three cylindroids corresponding to the distinct pairs of input screws. They have computed the principal pitches from the normal form of the hyperboloid. However, their approach involves solutions of (up to 9) simultaneous linear equations. Therefore it is procedural in nature, and as we show in section 5.2, the numerical results obtained by it can be highly inaccurate. Fang and Huang [7] have used the planar representation of a third-order screw system and the condition of 
degeneracy of the conic-sections in this plane to identify the principal screws. Huang and Wang [8] have used the above formulation in the context of a 3-RPS pyramid manipulator and identified numerically its principal screws at different configurations. Huang, Wang and Fang [9] have studied the 3-RPS manipulator proposed by Lee and Shah [10], and termed it a deficient-rank manipulator as the associated three-system is found to belong to the fourth or the seventh special systems discussed in [4]. Huang, Li and Zuo [11] have combined the concepts of reciprocal screws, planar degeneration etc. described above to analyse the feasible motions of a special 3-UPU manipulator.

In [12], Bandyopadhyay and Ghosal have proposed a new approach based on the eigenproblem of a symmetric dual matrix. The complete solution of the dual eigenproblem requires the solution of a generalised eigenproblem involving two real symmetric matrices (namely the real and dual parts of the dual matrix), and leads to the set of principal screws. In this paper, we apply this method to study the principal screws of general two-, three-, four-, and five-systems. We show that for two-, and three-systems, the generalised eigenproblem leads to a quadratic and a cubic equation respectively. Therefore a closed-form exact solution is always obtainable in these cases. We derive the expressions for the principal screws and their pitches in these cases. For the sake of completeness, we derive the classical results pertaining to the cylindroid and the hyperboloid within our formulation, and interpret some of the special screw systems algebraically. We derive algebraically the conditions defining some of the special two-, three-systems described in [4]. We also present the analysis of the four-, and five-systems, which differs from the analysis of the previous two. The novelty in our work is that unlike the other formulations, it does not require the computation of the reciprocal systems as a starting point.

The paper is organised as follows: in section 2, we present a brief description of the mathematical formulation of our approach for the general two-, three-systems. In section 3, some closed-from results pertaining to these are presented. The four-, and five-systems are discussed in section 4 . In section 5, we present four examples to illustrate our formulation. Finally, we present the conclusions in section 6 . 


\section{Mathematical Development}

In this section, we describe the formulation very briefly. We follow the same notations and formulation presented in [12], therefore the reader is directed to [12] for the details. For an introduction to dual numbers and their use in kinematics, the reader may please refer to $[13,14]$.

The forward linear velocity of a $n$-degrees-of-freedom manipulator may be written as

$$
\boldsymbol{v}=\boldsymbol{J}_{\boldsymbol{v}} \dot{\boldsymbol{\theta}}, \quad \dot{\boldsymbol{\theta}} \in \mathbb{R}^{n}
$$

where $\dot{\boldsymbol{\theta}}$ is the vector of joint rates, and $\boldsymbol{J}_{\boldsymbol{v}}$ the corresponding Jacobian. Similarly, the angular velocity is written as:

$$
\omega=J_{\omega} \dot{\theta}
$$

The algebra of dual numbers allow a combination of these two velocities into the dual velocity vector, $\hat{\mathcal{V}}$, as follows:

$$
\begin{aligned}
\hat{\mathcal{V}} & =\boldsymbol{\omega}+\epsilon \boldsymbol{v} \\
\Rightarrow \hat{\mathcal{V}} & =\boldsymbol{J}_{\boldsymbol{\omega}} \dot{\boldsymbol{\theta}}+\epsilon \boldsymbol{J}_{\boldsymbol{v}} \dot{\boldsymbol{\theta}} \\
\Rightarrow \hat{\mathcal{V}} & =\hat{\boldsymbol{J}} \dot{\boldsymbol{\theta}}
\end{aligned}
$$

where $\hat{\boldsymbol{J}}=\boldsymbol{J}_{\boldsymbol{\omega}}+\epsilon \boldsymbol{J}_{\boldsymbol{v}}$ is the dual Jacobian matrix.

The dual vector $\hat{\mathcal{V}}$ represents a twist associated with the first-order properties of the motion of a rigid body (see, e.g., [13]). In a spatial manipulator, the twists of the endeffector (the platform or the equivalent in case of a parallel manipulator) are of primary interest in terms of analysis and design. The dual Jacobian, $\hat{\boldsymbol{J}}$, is in general a function of the motion parameters, i.e., the joint variables in case of a manipulator. Therefore, the resulting twist varies not only with the joint rates $\dot{\boldsymbol{\theta}}$, but also with the configuration governed by $\boldsymbol{\theta}$. To decouple these two factors, we freeze the configuration, and obtain the set of all possible twists in that configuration. This technique is very well-known in manipulator kinematics, and has been applied in terms of the dual Jacobian in [12]. The main points of the formulation are reproduced below.

To study the distributions of twists, we obtain the extreme values of $\|\hat{\mathcal{V}}\|_{d}$ (the dual norm 
of $\hat{\mathcal{V}}$ ) under the constraint $\|\dot{\boldsymbol{\theta}}\|=1$. This results in the following dual eigenproblem:

$$
\begin{aligned}
& \hat{\boldsymbol{g}} \dot{\boldsymbol{\theta}}=\hat{\lambda} \dot{\boldsymbol{\theta}}, \text { where } \\
& \hat{\boldsymbol{g}}=\hat{\boldsymbol{J}}^{T} \hat{\boldsymbol{J}}
\end{aligned}
$$

Writing $\hat{\boldsymbol{g}}=\boldsymbol{g}+\epsilon \boldsymbol{g}_{0}, \boldsymbol{g}, \boldsymbol{g}_{0} \in \mathbb{R}^{n \times n}$, we get:

$$
\begin{aligned}
& \boldsymbol{g}+\epsilon \boldsymbol{g}_{0}=\hat{\boldsymbol{J}}^{T} \hat{\boldsymbol{J}} \\
\Rightarrow & \boldsymbol{g}+\epsilon \boldsymbol{g}_{0}=\boldsymbol{J}_{\boldsymbol{\omega}}^{T} \boldsymbol{J}_{\boldsymbol{\omega}}+\epsilon\left(\boldsymbol{J}_{\boldsymbol{\omega}}^{T} \boldsymbol{J}_{\boldsymbol{v}}+\boldsymbol{J}_{\boldsymbol{v}}^{T} \boldsymbol{J}_{\boldsymbol{\omega}}\right) \\
\Rightarrow & \boldsymbol{g}=\boldsymbol{J}_{\boldsymbol{\omega}}^{T} \boldsymbol{J}_{\boldsymbol{\omega}}, \text { and } \\
& \boldsymbol{g}_{0}=\boldsymbol{J}_{\boldsymbol{\omega}}^{T} \boldsymbol{J}_{\boldsymbol{v}}+\boldsymbol{J}_{\boldsymbol{v}}^{T} \boldsymbol{J}_{\boldsymbol{\omega}}
\end{aligned}
$$

Returning to the dual eigenproblem (4), we find it equivalent to the following pair of real eigenproblems:

$$
\begin{aligned}
& \boldsymbol{g} \dot{\boldsymbol{\theta}}=\lambda \dot{\boldsymbol{\theta}} \\
& \boldsymbol{g}_{0} \dot{\boldsymbol{\theta}}=\lambda_{0} \dot{\boldsymbol{\theta}}
\end{aligned}
$$

The two eigenproblems in equation (6) are consistent iff the matrices $\boldsymbol{g}$ and $\boldsymbol{g}_{0}$ share the eigenvector $\dot{\boldsymbol{\theta}}$. From linear algebra, the condition implies that:

$$
\boldsymbol{g} \boldsymbol{g}_{0}=\boldsymbol{g}_{0} \boldsymbol{g} \Leftrightarrow\left[\boldsymbol{g}, \boldsymbol{g}_{0}\right]=\mathbf{0}
$$

In general, this condition is not satisfied automatically. However, if $\boldsymbol{g}$ is positive definite, there exists a transformation $\boldsymbol{T}$ of $\mathbb{R}^{n}$, which reduces $\boldsymbol{g}$ and $\boldsymbol{g}_{0}$ to such forms that they commute. The matrix $\boldsymbol{g}$ can be positive definite only if $n$ equals 1,2 , or 3 . Neglecting the trivial case of one-DOF motion, this refers to the general two-, and three-systems. A three-step method to obtain $\boldsymbol{T}$ and their geometric significance are described in [12]. The same result can also be obtained by direct algebraic manipulation as follows. Since $\lambda \neq 0$ under the assumptions, we can write from equations (6):

$$
\begin{aligned}
& \boldsymbol{g}_{0} \dot{\boldsymbol{\theta}}=\frac{\lambda_{0}}{\lambda} \boldsymbol{g} \dot{\boldsymbol{\theta}} \\
\Rightarrow & \left(\boldsymbol{g}_{0}-\frac{\lambda_{0}}{\lambda} \boldsymbol{g}\right) \dot{\boldsymbol{\theta}}=\mathbf{0}, \quad \lambda \neq 0
\end{aligned}
$$

The above equation represents a generalised eigenvalue problem of $\boldsymbol{g}_{0}$ with respect to $\boldsymbol{g}$. It has been shown in [12] that the generalised eigenvalues, $\frac{\lambda_{0}}{\lambda}$, are double the principal pitches 
(denoted by $h^{h}$ ). Denoting the $i$ th generalised eigenvector by $\dot{\boldsymbol{\theta}}_{i}^{h}$, we can rewrite equation (8) as:

$$
\left(\boldsymbol{g}_{0}-2 h_{i}^{h} \boldsymbol{g}\right) \dot{\boldsymbol{\theta}}_{i}^{h}=\mathbf{0}
$$

The generalised eigenvalues, $h_{i}^{h}$, can be obtained from the generalised characteristic equation:

$$
\operatorname{det}\left(\boldsymbol{g}_{0}-2 h_{i}^{h} \boldsymbol{g}\right)=0
$$

The eigenvectors, $\dot{\boldsymbol{\theta}}_{i}^{h}$, form the columns of the required transformation $\boldsymbol{T}$. The principal screws are obtained from them in two steps. First, we obtain the principal twists by mapping the eigenvectors by $\hat{\boldsymbol{J}}$ (see equation (3)):

$$
\hat{\mathcal{V}}_{i}^{h}=\hat{\boldsymbol{J}} \dot{\boldsymbol{\theta}}_{i}^{h}
$$

Next, we normalise the principal twists to obtain the principal screws, $\hat{\$}_{i}^{h}$ :

$$
\hat{\S}_{i}^{h}=\hat{\mathcal{V}}_{i}^{h} / \operatorname{real}\left(\left\|\hat{\mathcal{V}}_{i}^{h}\right\|_{d}\right)
$$

It may be noted that after the transformation, $\hat{\boldsymbol{g}}$ is diagonalised, i.e., the dual inner-product of two non-identical principal screws vanish:

$$
\left\langle\hat{\aleph}_{i}^{h}, \hat{\$}_{j}^{h}\right\rangle_{d}=0+\epsilon 0 \Leftrightarrow i \neq j
$$

From the definition of inner product of screws (see, e.g., [13]) it follows immediately that the principal screws meet at one point in space orthogonally. The pitches along these screws, being the double of the generalised eigenvalues of $\boldsymbol{g}_{0}$ with respect to $\boldsymbol{g}$, are also the extreme values of the pitch ${ }^{1}$. Therefore, the screws $\hat{\$}_{i}^{h}, i=1,2$ or $1,2,3$, are indeed the principal screws described by Ball [3] and Hunt [4]. We term the set of screws the $h$-basis of $\mathbb{P}^{5}[12]$. This approach is novel to the best of our knowledge, and it seems to have the following advantages over existing work:

- The problem of identification of the principal screws is reduced to a generalised eigenproblem, which is well-studied in literature (see, e.g., [15]). Therefore, the properties of screw systems can be analysed using standard algebraic concepts.

\footnotetext{
${ }^{1} \mathrm{~A}$ more direct proof of this claim is presented in Appendix A.
} 
- The conditions for a generic screw system to reduce to the special cases (as listed in [4]) can be obtained symbolically in closed-form, in terms of the coefficients of the generalised characteristic polynomial corresponding to equation (9).

- For three-DOF rigid-body motion, sizes of $\boldsymbol{g}$ and $\boldsymbol{g}_{0}$ are limited to $3 \times 3$, and hence the generalised characteristic polynomial corresponding to equation (9) is restricted to a cubic at the most. Therefore, we can solve the problem in closed form. Further, since $\boldsymbol{g}$ is positive-definite in this case, we are also guaranteed to get real eigenvalues and eigenvectors (see, e.g., [15]).

We present below the closed-form results for two-, and three-systems. The one-system is trivial, as its only constituent screw serves as the principal screw.

\section{Analysis of Two-, Three-systems in the $h$-basis}

In this section, we present the symbolic expressions for the principal screws of $h$-basis in terms of the input screw parameters. These exact expressions represent new contributions of this paper.

We use the notation $c_{\theta}, s_{\theta}$ respectively for $\cos \theta$ and $\sin \theta$ etc. The perpendicular distance

between the screws $\hat{\$}_{i}$ and $\hat{\$}_{j}$ is denoted by $d_{i j}$ and the angle is denoted by $\alpha_{i j}$. Further, $c_{i}$ and $s_{i}$ denote $\cos \theta_{i}$ and $\sin \theta_{i}$ respectively, and $c_{i j}, s_{i j}$ denote $\cos \alpha_{i j}$ and $\sin \alpha_{i j}$ respectively.

\subsection{Two-system}

We first derive the closed-form expressions of the principal screws. We then recover the classical equation of the cylindroid associated with the general two-system using the dual algebra formulation. We also present a few special cases within the two-system. 


\subsubsection{Derivation of the $h$-basis}

In this case, the input screws are denoted by $\hat{\$}_{1}$ of pitch $h_{1}$ and $\hat{\$}_{2}$ of pitch $h_{2}$. The generalised characteristic equation is a quadratic of the form:

$$
\begin{aligned}
& a_{0} h^{2}+a_{1} h+a_{2}=0, \text { where } \\
& a_{0}=4 \operatorname{det}(\boldsymbol{g})=4 s_{12}^{2} \\
& a_{1}=4 s_{12}\left(d_{12} c_{12}+\left(h_{1}+h_{2}\right) s_{12}\right) \\
& a_{2}=\operatorname{det}\left(\boldsymbol{g}_{0}\right)=4 h_{1} h_{2}-\left(h_{1}+h_{2}\right)^{2} c_{12}^{2}-d_{12}^{2} s_{12}^{2}+d_{12} s_{12}\left(h_{1}+h_{2}\right)
\end{aligned}
$$

This equation may be solved to obtain the principal pitches as:

$$
\begin{array}{ll}
h_{1}^{h}=\left(h_{1}+h_{2}\right)+d_{12} \frac{c_{12}}{s_{12}}-\frac{D}{s_{12}^{2}}, & s_{12} \neq 0 \\
h_{2}^{h}=\left(h_{1}+h_{2}\right)+d_{12} \frac{c_{12}}{s_{12}}+\frac{D}{s_{12}^{2}}, \quad D=\sqrt{d_{12}^{2}+\left(h_{1}-h_{2}\right)^{2}}
\end{array}
$$

The (non-normalised) eigenvectors are given by:

$$
\begin{aligned}
& \dot{\boldsymbol{\theta}}_{1}^{h}=\left(h_{2}-h_{1}+D s_{12},\left(h_{1}-h_{2}\right) c_{12}+d_{12} s_{12}\right)^{T} \\
& \dot{\boldsymbol{\theta}}_{2}^{h}=\left(h_{2}-h_{1}-D s_{12},\left(h_{1}-h_{2}\right) c_{12}+d_{12} s_{12}\right)^{T}
\end{aligned}
$$

The corresponding principal screws are obtained by mapping the eigenvectors by $\hat{\boldsymbol{J}}$ and normalising the result (as in equations $(11,12)$ ). The principal screws intersect orthogonally at a point in $\mathbb{R}^{3}$. We translate the origin to this point, and align the new coordinate axes along $\boldsymbol{q}_{1}, \boldsymbol{q}_{2}, \boldsymbol{q}_{1} \times \boldsymbol{q}_{2}$, where $\boldsymbol{q}_{1}, \boldsymbol{q}_{2}$ are parallel to $\hat{\$}_{1}^{h}, \hat{\$}_{2}^{h}$ respectively. In this new frame, principal screws attain their simplest forms:

$$
\hat{\$}_{i}^{h}=\boldsymbol{e}_{i}\left(1+\epsilon h_{i}^{h}\right), i=1,2
$$

where $\left\{\boldsymbol{e}_{i}\right\}_{i=1}^{3}$ denotes the standard basis of $\mathbb{R}^{3}$.

\subsubsection{Derivation of the cylindroid}

A general screw in a two-system can be expressed as a one-parameter system in the parameter $\theta \in[0,2 \pi]:$

$$
\begin{aligned}
\hat{\boldsymbol{\$}} & =\hat{\boldsymbol{\$}}_{1}^{h} c_{\theta}+\hat{\boldsymbol{\$}}_{2}^{h} s_{\theta} \\
& =c_{\theta} \boldsymbol{e}_{1}+s_{\theta} \boldsymbol{e}_{2}+\epsilon\left(h_{1}^{h} c_{\theta} \boldsymbol{e}_{1}+h_{2}^{h} s_{\theta} \boldsymbol{e}_{2}\right)
\end{aligned}
$$


Writing $\hat{\boldsymbol{\$}}$ as $\boldsymbol{s}+\epsilon \boldsymbol{s}_{0}$, and noting that $\|\boldsymbol{s}\|=1$, the foot of the perpendicular from the origin may be found as: $\boldsymbol{r}_{0}=\boldsymbol{s} \times \boldsymbol{s}_{0}=\left(0,0,\left(h_{2}^{h}-h_{1}^{h}\right) s_{\theta} c_{\theta}\right)^{T}$. Denoting $\left(h_{2}^{h}-h_{1}^{h}\right) s_{\theta} c_{\theta}$ by $z$ and writing $x=r \cos \theta, y=r \sin \theta, r \in \mathbb{R}^{+}$, we find:

$$
z=\left(h_{2}^{h}-h_{1}^{h}\right) \frac{x y}{r^{2}}=\left(h_{2}^{h}-h_{1}^{h}\right) \frac{x y}{x^{2}+y^{2}}
$$

Rationalising, we arrive at the well-known equation of the cylindroid [3, 4]:

$$
z\left(x^{2}+y^{2}\right)+\left(h_{1}^{h}-h_{2}^{h}\right) x y=0
$$

\subsubsection{Algebraic analysis of the special cases}

One advantage of the algebraic formulation is that the conditions leading to the special cases of screw systems can be obtained in terms of closed-form algebraic equations.

Equal pitches (finite): The condition for the pitches to be the same is obtained by setting the discriminant of equation (14) to zero:

$$
16\left(d_{12}^{2}+\left(h_{1}-h_{2}\right)^{2}\right) s_{12}^{2}=0
$$

The last equation shows that the pitches are equal if either of the two conditions hold:

1. $s_{12}=0$ : Input screws are coaxial.

2. $d_{12}=0=h_{1}-h_{2}$ : The input screw axes intersect, and the screws have the same pitch.

However, condition 1 above implies that the screws have infinite pitch, since $a_{0}=4 \operatorname{det}(\boldsymbol{g})=4 s_{12}^{2}$, hence only condition 2 gives finite, equal pitches. This defines a one-parameter family of screws, the free parameter being the angle between the axes of the screws.

Infinite pitches: From the above expressions of $h_{1}^{h}$ and $h_{2}^{h}$, both the pitches become infinite when $s_{12}=0$, i.e., the screws are coaxial.

\subsection{Three-system}

We start with the closed-form results for the general three-system, and then derive the classical equation of the pitch-hyperboloid describing the distribution of pitches. The algebraic treatment of a few special cases described by Hunt [4] is also presented. 


\subsubsection{Derivation of the $h$-basis}

The generalised characteristic equation (10) is a cubic in this case:

$$
a_{0} h^{3}+a_{1} h^{2}+a_{2} h+a_{3}=0
$$

Closed-form expressions of the coefficients are computed using the symbolic simplification algorithms presented in [16]:

$$
\begin{aligned}
a_{0}= & -8 \operatorname{det}(\boldsymbol{g}) \\
& =4\left(1+A_{1}-4 A_{2}\right) \\
a_{1}= & -H\left(1+A_{1}-4 A_{2}\right) \\
& \quad+8\left(\left(c_{12}-c_{23} c_{31}\right) d_{12} s_{12}+\left(c_{23}-c_{12} c_{31}\right) d_{23} s_{23}+\left(c_{31}-c_{12} c_{23}\right) d_{31} s_{31}\right) \\
& \quad h_{1}^{2}\left(2+A_{1}-2 \cos \left(2 \alpha_{23}\right)\right)+h_{2}^{2}\left(2+A_{2}-2 \cos \left(2 \alpha_{31}\right)\right)+h_{3}^{2}\left(2+A_{1}-2 \cos \left(2 \alpha_{12}\right)\right) \\
& +2 h_{1} h_{2}\left(2 A_{1}-6 A_{2}-1-\cos \left(2 \alpha_{12}\right)\right)+2 h_{2} h_{3}\left(2 A_{1}-6 A_{2}-1-\cos \left(2 \alpha_{23}\right)\right) \\
& +2 h_{3} h_{1}\left(2 A_{1}-6 A_{2}-1-\cos \left(2 \alpha_{31}\right)\right) \\
- & 4 h_{1}\left(\left(c_{12}-c_{23} c_{31}\right) d_{12} s_{12}+2\left(c_{23}-c_{12} c_{31}\right) d_{23} s_{23}+\left(c_{31}-c_{12} c_{23}\right) d_{31} s_{31}\right) \\
- & 4 h_{2}\left(\left(c_{12}-c_{23} c_{31}\right) d_{12} s_{12}+\left(c_{23}-c_{12} c_{31}\right) d_{23} s_{23}+2\left(c_{12} c_{23}-c_{31}\right) d_{31} s_{31}\right) \\
- & 4 h_{3}\left(2\left(c_{12}-c_{23} c_{31}\right) d_{12} s_{12}+\left(c_{23}-c_{12} c_{31}\right) d_{23} s_{23}+\left(c_{12} c_{23}-c_{31}\right) d_{31} s_{31}\right) \\
+ & 2 d_{12}^{2} s_{12}^{2}+2 d_{23}^{2} s_{23}^{2}+2 d_{31}^{2} s_{31}^{2} \\
- & 4\left(d_{12} d_{23} s_{12} s_{23} c_{31}+d_{23} d_{31} s_{31} s_{23} c_{12}+d_{12} d_{31} s_{12} s_{31} c_{23}\right)
\end{aligned}
$$




$$
\begin{aligned}
a_{3}= & \operatorname{det}\left(\boldsymbol{g}_{0}\right) \\
= & 2\left(c_{12} c_{23}-c_{31}\right) c_{31} h_{1}^{2} h_{2}-2 c_{12}\left(c_{12}-c_{23} c_{31}\right) h_{1}^{2} h_{3}-2 c_{12} c_{31} d_{23} s_{23} h_{1}^{2} \\
& -2 c_{23}\left(c_{23}-c_{12} c_{31}\right) h_{1} h_{2}^{2}-2\left(A_{1}-2 A_{2}-1\right) h_{1} h_{2} h_{3} \\
& -\left(2 c_{23} c_{31} d_{12} s_{12}+2\left(2 c_{23}-c_{12} c_{31}\right) d_{23} s_{23}-2\left(c_{12} c_{23}-2 c_{31}\right) d_{31} s_{31}\right) h_{1} h_{2} \\
& -2 c_{23}\left(c_{23}-c_{12} c_{31}\right) h_{1} h_{3}^{2} \\
& +\left(2\left(2 c_{12}-c_{23} c_{31}\right) d_{12} s_{12}+2\left(2 c_{23}-c_{12} c_{31}\right) d_{23} s_{23}-2 c_{12} c_{23} d_{31} s_{31}\right) h_{1} h_{3} \\
& -\left(2 d_{23}^{2} s_{23}^{2}+2 c_{31} d_{12} d_{23} s_{12} s_{23}+2 c_{12} d_{23} d_{31} s_{31} s_{23}\right) h_{1} \\
& -2 c_{12}\left(c_{12}-c_{23} c_{31}\right) h_{2}^{2} h_{3}-2 c_{12} c_{23} d_{31} s_{31} h_{2}^{2}+2\left(c_{12} c_{23}-c_{31}\right) c_{31} h_{2} h_{3}^{2} \\
& +\left(2 d_{12} s_{12}\left(c_{12}-c_{23} c_{31}\right)-2 c_{12} c_{31} d_{23} s_{23}-2\left(c_{12} c_{23}-2 c_{31}\right) d_{31} s_{31}\right) h_{2} h_{3} \\
& -\left(2 d_{31}^{2} s_{31}^{2}+2 c_{23} d_{12} d_{31} s_{12} s_{31}+2 c_{12} d_{23} d_{31} s_{23} s_{31}\right) h_{2}-2 c_{23} c_{31} d_{12} s_{12} h_{3}^{2} \\
& -\left(2 d_{12}^{2} s_{12}^{2}+2 c_{31} d_{12} d_{23} s_{23} s_{12}+2 c_{23} d_{12} d_{31} s_{31} s_{12}\right) h_{3}-2 d_{12} d_{23} d_{31} s_{12} s_{23} s_{31}
\end{aligned}
$$

where $A_{1}=\cos 2 \phi_{12}+\cos 2 \phi_{23}+\cos 2 \phi_{31}, A_{2}=c_{12} c_{23} c_{31}$ and $H=h_{1}+h_{2}+h_{3}$. Equation (19) admits only real solutions, as it arises from the simultaneous diagonalisation of two quadratic forms, $\boldsymbol{g}$ and $\boldsymbol{g}_{0}$, where $\boldsymbol{g}$ is positive definite (see, e.g., [15]). The generalised eigenvectors (non-normalised) corresponding to $h_{i}^{h}$ may be obtained as:

$$
\begin{gathered}
\dot{\boldsymbol{\theta}}_{i}^{h}=\left(n_{1}, n_{2}, n_{3}\right)^{T} \text {, where } \\
n_{1}=2 c_{31}\left(h_{i}^{h}-h_{2}\right)\left(2 h_{i}^{h}-h_{1}-h_{3}\right)-\left(c_{12}\left(2 h_{i}^{h}-h_{1}-h_{2}\right)+d_{12} s_{12}\right) \\
\quad \times\left(c_{23}\left(2 h_{i}^{h}-h_{2}-h_{3}\right)+d_{23} s_{23}\right)+2 d_{31}\left(h_{i}^{h}-h_{2}\right) s_{31} \\
n_{2}=2 c_{23}\left(h_{i}^{h}-h_{1}\right)\left(2 h_{i}^{h}-h_{2}-h_{3}\right)+2 d_{23}\left(h_{i}^{h}-h_{1}\right) s_{23}-\left(c_{12}\left(2 h_{i}^{h}-h_{1}-h_{2}\right)+d_{12} s_{12}\right) \\
\\
\quad \times\left(c_{31}\left(2 h_{i}^{h}-h_{1}-h_{3}\right)+d_{31} s_{31}\right) \\
\\
n_{3}=c_{12}^{2}\left(h_{1}+h_{2}\right)^{2}+\left(d_{12}^{2}+4 h_{i}^{h}\left(-h_{i}^{h}+h_{1}+h_{2}\right)\right) s_{12}^{2}-4 h_{1} h_{2}-\sin \left(2 \alpha_{12}\right) d_{12}\left(-2 h_{i}^{h}+h_{1}+h_{2}\right)
\end{gathered}
$$$$
\text { The corresponding principal screws are obtained from equations }(11,12) \text {. }
$$

\subsubsection{Derivation of the pitch-hyperboloid}

As in the case of two-system, we move the origin to the point of concurrence of the principal screws, and align the coordinate axes along the principal screw axes to obtain a new reference 
frame of $\mathbb{R}^{3}$, in which the principal screws have the form $\hat{\$}_{i}=\boldsymbol{e}_{i}\left(1+\epsilon h_{i}^{h}\right), i=1,2,3$. Under the unit speed constraint, the possible screws can be written as a two-parameter family:

$$
\begin{aligned}
\hat{\$} & =\hat{\$}_{1} l+\hat{\$}_{2} m+\hat{\$}_{3} n \\
& =l \boldsymbol{e}_{1}+m \boldsymbol{e}_{2}+n \boldsymbol{e}_{3}+\epsilon\left(l h_{1}^{h} \boldsymbol{e}_{1}+m h_{2}^{h} \boldsymbol{e}_{2}+n h_{3}^{h} \boldsymbol{e}_{3}\right)
\end{aligned}
$$

where $l, m, n \in \mathbb{R}, l^{2}+m^{2}+n^{2}=1$. The foot of the perpendicular from the origin to the axis of $\hat{\$}$ may be found as:

$$
\begin{aligned}
\boldsymbol{r}_{0} & =\boldsymbol{s} \times \boldsymbol{s}_{0} \\
& =\left(\left(h_{3}^{h}-h_{2}^{h}\right) m n,\left(h_{1}^{h}-h_{3}^{h}\right) \ln ,\left(h_{2}^{h}-h_{1}^{h}\right) l m\right)^{T}
\end{aligned}
$$

By setting to zero $l, m$ and $n$ in turn, we obtain the cylindroids with the nodal axes along $\boldsymbol{e}_{1}, \boldsymbol{e}_{2}$, and $\boldsymbol{e}_{3}$ respectively. Therefore we arrive at the result obtained in [3, 4], that for an arbitrary three-DOF motion, the resultant screw axis lies on the intersection of three cylindroids concurrent at a point, with their nodal axes mutually orthogonal. We now derive the equation of the hyperboloid which describes the screw distribution completely [3, 4]. Let the screw axis pass through the point $(x, y, z)^{T}$. Hence its moment about the origin is given by $\boldsymbol{q}_{0}=(x, y, z) \times \boldsymbol{q}$. However, from the definitions, $\boldsymbol{q}_{0}=\boldsymbol{s}_{0}-h \boldsymbol{q}$. Therefore we have the equation:

$$
(x, y, z)^{T} \times \boldsymbol{q}=\boldsymbol{s}_{0}-h \boldsymbol{q}
$$

Expanding this equation and rearranging, we obtain

$$
\left(\begin{array}{ccc}
h-h_{1}^{h} & -z & y \\
z & h-h_{2}^{h} & -x \\
-y & x & h-h_{3}^{h}
\end{array}\right)\left(\begin{array}{l}
l \\
m \\
n
\end{array}\right)=0
$$

For the above homogeneous equations to have a non-trivial solution, we must have the determinant of the matrix on the left hand side as zero. This condition yields:

$$
x^{2}\left(h-h_{1}^{h}\right)+y^{2}\left(h-h_{2}^{h}\right)+z^{2}\left(h-h_{3}^{h}\right)+\left(h-h_{1}^{h}\right)\left(h-h_{2}^{h}\right)\left(h-h_{3}^{h}\right)=0
$$

The above equation gives the pitch $h$ associated with a line passing through any arbitrary point $(x, y, z)$, and corroborates with the results obtained by Hunt [4]. 


\subsubsection{Algebraic analysis of the special cases}

We now derive the conditions defining some of the special three-systems described by Hunt [4]. Two pitches are equal (all finite): We note that all the principal pitches are finite if $a_{0} \neq 0$ in equation (19). To find the condition that the two of the principal pitches are the same, we scale the equation (19) by $a_{0}$ and compare with the equation:

$$
(x-\alpha)^{2}(x-\beta)=0
$$

where $\alpha, \beta$ represent the repeated, and the non-repeated roots respectively. Equating the corresponding coefficients in equations $(19,23)$, we obtain the following set of equations:

$$
\begin{array}{r}
2 \alpha+\beta+\frac{a_{1}}{a_{0}}=0 \\
\alpha^{2} \beta+\frac{a_{3}}{a_{0}}=0 \\
\alpha^{2}+2 \alpha \beta-\frac{a_{2}}{a_{0}}=0
\end{array}
$$

Eliminating $\alpha, \beta$ from the above three equations, we obtain the required condition as:

$$
4 a_{1}^{3} a_{3}-a_{1}^{2} a_{2}^{2}-18 a_{0} a_{1} a_{2} a_{3}+a_{0}\left(4 a_{2}^{3}+27 a_{0} a_{3}^{2}\right)=0
$$

All pitches are equal (all finite): All the pitches are equal when the coefficients of the standard form of equation (19) vanish. In terms of the coefficients of the original equation, the required conditions are:

$$
\begin{aligned}
2 a_{1}^{3}-9 a_{0} a_{1} a_{2}+27 a_{0}^{2} a_{3}^{2} & =0 \\
a_{1}^{2}-3 a_{0} a_{2} & =0, \quad a_{0} \neq 0
\end{aligned}
$$

One pitch infinite, two pitches unequal (finite): To derive this set of conditions, we rewrite equation (19) in terms of $\sigma=1 / h$, to obtain:

$$
a_{3} \sigma^{3}+a_{2} \sigma^{2}+a_{1} \sigma+a_{0}=0
$$

The condition for one of the pitches being infinite is equivalent to the vanishing of one of the $\sigma$ 's, i.e.,

$$
a_{0}=0, \quad a_{1}, a_{2}, a_{3} \text { are not all zero }
$$


Further, the two finite pitches are unequal, i.e., discriminant of the residual quadratic equation is nonzero:

$$
a_{2}^{2}-4 a_{1} a_{3} \neq 0
$$

One pitch infinite, two pitches equal (finite): This case is similar to the last one, and the conditions are given by:

$$
\begin{aligned}
a_{0} & =0 \\
a_{2}^{2}-4 a_{1} a_{3} & =0, \quad a_{1}, a_{2}, a_{3} \text { are not all zero }
\end{aligned}
$$

Two pitches infinite, one finite: From equation (27), the conditions for this case can be obtained as:

$$
a_{0}=a_{1}=0, a_{2}, a_{3} \neq 0
$$

All pitches infinite: From equation (27), the conditions for this case can be obtained as:

$$
a_{0}=a_{1}=a_{2}=0, a_{3} \neq 0
$$

\section{Analysis of Four-, Five-systems in the $h$-basis}

We now study the cases where $\boldsymbol{g}$ is positive semi-definite. The formulation differs from the two-, and three-systems in that the generalised eigenproblem formulation is not possible in these cases, as these are characterised by $\operatorname{det}(\boldsymbol{g})=0$. Traditionally, in such cases the reciprocal screw systems are computed first, and their properties are analysed $[3,4]$. However, we continue to use the same criteria for the identification of the principal screws- namely the extreme values of the pitch. Using the concept of degrees-of-freedom partitioning described in [12], we partition the space of input screws into two sets, having finite and infinite pitches respectively. We then find the principal screws of the former set. These screws are reciprocal to the principal screws of the reciprocal system by construction, and therefore we need not compute the reciprocal screw system explicitly as an intermediate step in our formulation. The basis can be completed by the addition of two coaxial screws of infinite pitches in accordance with the concept of classical co-reciprocal basis [4]. The steps involved for the analysis of four-system varies slightly from the five-system case, and we describe both of them in details below. 


\section{$4.1 \quad$ Four-systems}

We study below the generic case considered in [3, 4]. The four-system considered constitutes of three screws of finite pitch and one of infinite pitch, signifying one pure translational DOF. The steps of computation may be delineated as follows.

- Identify the pure translational twists: We use the concept of partitioning of degrees-of-freedom described in [12] to find out the screw representing the pure translation. This is done by first obtaining the nullspace of $\boldsymbol{g}$, and then using it to obtain the screw $\hat{\$}_{T}$, which carries all the translational twists:

$$
\hat{\boldsymbol{\$}}_{T}=\hat{\boldsymbol{J}} \dot{\boldsymbol{\theta}}^{n}, \quad \boldsymbol{g} \dot{\boldsymbol{\theta}}^{n}=\mathbf{0}
$$

There are three linearly independent screws having finite pitches, which lie in the

column-space of $\hat{\boldsymbol{J}}$. We denote these screws by $\hat{\$}_{C i}, i=1,2,3$, and their collection by $\hat{\$}_{C}$. Note that the original four-system is a direct sum of $\hat{\$}_{T}$ and $\hat{\$}_{C}$.

- Decompose $\hat{\$}_{C}$ in sets of screws parallel and perpendicular to $\hat{\$}_{T}$ : It is known in literature, that the principal basis in this case consists of two coaxial screws of infinite pitch, and two screws of finite pitch orthogonal to this pair. In other words there is a two-parameter family of screws having finite pitch, which are orthogonal to the direction of pure translation. To find this set, we now decompose $\hat{\$}_{C}$ into two disjoint subspaces, one of which have the axes parallel to the direction of $\hat{\$}_{T}$, and the other perpendicular to it. This is done by constructing a general screw, $\hat{\$}_{c}$, within the space $\hat{\$}_{C}$ by a linear combination of $\hat{\$}_{C i}$ :

$$
\hat{\$}_{c}=c_{i} \hat{\$}_{C i}, \quad c_{i} \in \mathbb{R}, i=1,2,3
$$

We are interested in the solutions for $c_{i}$ such that the following orthogonality condition is satisfied:

$$
\operatorname{real}\left(\left\langle\hat{\$}_{\mathrm{C}}, \hat{\$}_{\mathrm{T}}\right\rangle_{\mathrm{d}}\right)=0
$$

Further, we introduce the normalisation condition to confine $\hat{\$}_{c}$ to $\mathbb{P}^{5}$ (i.e., to ensure $\left.\operatorname{real}\left(\left\|\hat{\$}_{c}\right\|_{d}\right)=1\right)$ :

$$
c_{1}^{2}+c_{2}^{2}+c_{3}^{2}=1
$$


Equations $(35,36)$ define two constraints on $c_{i}$. There is a freedom in choosing one of the parameters, and without loss of generality, we choose $c_{3}$ arbitrarily. For each such choice of $c_{3}$, equations $(35,36)$ yield two (generally distinct) pairs of solutions for the other interpolation coefficients $c_{1}, c_{2}$. Each of these pairs lead to a pair of screws in $\hat{\$}_{C}$ via equation (34), which are perpendicular to $\hat{\$}_{T}$. We note that by the above construction, all the screws generated by linear combinations of these two screws are orthogonal to $\hat{\$}_{T}$, and they can have only finite pitch.

- Find the principal screws of the two-screw system formed above: Using the formulation for the two-system, we find the principal screws, $\hat{\$}_{1}^{h}$ and $\hat{\$}_{2}^{h}$, of the screwsystem formed in the previous step. Note that these two screws will be concurrent and mutually orthogonal, and they have the maximum and minimum pitch among the finite pitch screws of the original system. Therefore they form a part of the set of principal screws of the original system.

- Complete the principal basis using the co-reciprocal convention: The other two principal screws can have infinite pitch, hence by the above construction, they are restricted to be perpendicular to $\hat{\$}_{1}^{h}, \hat{\$}_{2}^{h}$. The location of these screws can be arbitrary. However, as per the definition of co-reciprocal basis [3], we choose these two coaxial screws to pass through the point of intersection of $\hat{\$}_{1}^{h}$ and $\hat{\$}_{2}^{h}$, with their axes parallel to that of $\hat{\$}_{T}$. The pitches of these two screws can be denoted as $\pm h_{\gamma}, h_{\gamma} \in[-\infty, \infty]$.

- Position the origin of the principal basis at the centre of the reciprocal cylindroid: The two-screw system generated by the screws $\hat{\$}_{1}^{h}, \hat{\$}_{2}^{h}$ formed in the above steps is not guaranteed to be reciprocal to the reciprocal of the original four-system, and the reciprocity theorem is not used in their construction. On the contrary, it may be shown that the $h$-basis formed thus is actually a two-parameter system, formed by the arbitrary translations of the cylindroid formed by $\hat{\$}_{1}^{h}, \hat{\$}_{2}^{h}$ in a plane perpendicular to the axis of $\hat{\$}_{T}$. This is possible since translations do not affect the pitch, or direction of the screws. The reciprocal basis of the original system is located at a particular point in this plane. This location, denoted by $\boldsymbol{O}^{h}(x, y, z)$, may be uniquely determined by using the fact that the two-system of reciprocal screws are reciprocal to the original four-system. The steps of the procedure are described below. 
1. Find the axes $\boldsymbol{q}_{1}^{h}, \boldsymbol{q}_{2}^{h}$ of the screws $\hat{\$}_{1}^{h}, \hat{\$}_{2}^{h}$ respectively. Denote their pitches by $h_{1}^{h}, h_{2}^{h}$ respectively.

2. Construct two screws, $\hat{\$}_{r 1}$ and $\hat{\$}_{r 2}$ coaxial with $\hat{\$}_{1}^{h}$ and $\hat{\$}_{2}^{h}$ respectively. Let their pitches be $\left(-h_{1}^{h}\right)$ and $\left(-h_{2}^{h}\right)$ respectively, and let them pass through the point $\boldsymbol{O}^{h}(x, y, z)$ :

$$
\begin{aligned}
& \hat{\boldsymbol{\$}}_{r 1}=\boldsymbol{q}_{1}^{h}+\epsilon\left(h_{1}^{h} \boldsymbol{q}_{1}^{h}+\boldsymbol{O}^{h}(x, y, z) \times \boldsymbol{q}_{1}^{h}\right) \\
& \hat{\boldsymbol{\$}}_{r 2}=\boldsymbol{q}_{2}^{h}+\epsilon\left(h_{2}^{h} \boldsymbol{q}_{2}^{h}+\boldsymbol{O}^{h}(x, y, z) \times \boldsymbol{q}_{2}^{h}\right)
\end{aligned}
$$

3. Use the reciprocal product (denoted by $\langle\cdot, \cdot\rangle_{r}$ ) to generate three equations in $x, y, z$ :

$$
\left\langle\hat{\$}_{r i}, \hat{\$}_{j}\right\rangle_{r}=0
$$

where $\hat{\$}_{j}$ is an element of the input four-system. Choose $(i, j)$ such that the equations are distinct.

4. Solve the above set of equations linearly for $(x, y, z)$.

Thus, in the process of locating the origin of the $h$-basis for the four-system uniquely, we locate the origin of the reciprocal two-system as well. The other screw parameters, such as the pitches, and the axes are obtained without using the reciprocal relationship. This final step only completes the determination of the reciprocal basis of the original four-system.

The above process is illustrated, and numerically verified for an example of a four-system in section 5 .

\section{$4.2 \quad$ Five-systems}

The analysis of the five-system is not very different from the above, hence we describe it briefly below.

The starting point, once again, is the decomposition of the input screws into finite-, and infinite-pitch subsets. In this case, in addition to the three principal screws of finite pitch, there are two pure translation screws, i.e., all screws of infinite pitch are parallel to a plane in this case, as opposed to a line in the above case. We identify the screws of finite pitch as those perpendicular to this plane. Since there can be only one such direction, the finite pitch 
is also fixed for all the screws in that direction. Therefore all the screws perpendicular to the translation screws qualify as one element of the principal basis of the four-system. The other four principal screws of the $h$-basis are determined by constructing two pairs of co-reciprocal screws of indeterminate pitch. Finally, the reciprocal basis of the original screw system may be found by locating the single principal screw in a plane, as in the case of four-system. We note that the computations involved in the cases of four- and five-systems include solutions of linear and quadratic equations, in addition to the eigenproblem in the first case. However, since all the computations can be done symbolically, the final results can still be obtained in closed form.

We note that the general six-system associated with six-DOF rigid-body motion spans $\mathbb{P}^{5}$ completely, and as such it has no constraints [4]. The screws can have any pitch in $[\infty, \infty]$, and their axes can have any direction and location in $\mathbb{R}^{3}$. Therefore analysis in the $h$-basis can yield no information about such motions.

\section{$5 \quad$ Illustrative Examples}

In this section, we illustrate, in closed form, some of the theoretical developments presented above with the example of a $3-\mathrm{R}$ serial manipulator and a general three-system studied by Zhang and $\mathrm{Xu}$ in [6]. We also demonstrate our algorithm for the four-system numerically. Finally, we analyse the instantaneous kinematics of an existing spatial parallel manipulator, namely the 3-RPS, using our method.

\subsection{Spatial 3-R manipulator}

The DH parameters of the manipulator are given in table 1 .

\begin{tabular}{|l|l|l|l|l|}
\hline$i$ & $\alpha_{i-1}$ & $a_{i-1}$ & $d_{i}$ & $\theta_{i}$ \\
\hline 1 & 0 & 0 & $d_{1}$ & $\theta_{1}$ \\
\hline 2 & $\alpha_{12}$ & $a_{12}$ & $d_{2}$ & $\theta_{2}$ \\
\hline 3 & $\alpha_{23}$ & $a_{23}$ & $d_{3}$ & $\theta_{3}$ \\
\hline
\end{tabular}

Table 1: DH parameters of the spatial 3-R manipulator 
The matrix $\boldsymbol{g}$ is computed as:

$$
\boldsymbol{g}=\left(\begin{array}{lll}
1 & c_{12} & c_{12} c_{23}-c_{2} s_{12} s_{23} \\
c_{12} & 1 & c_{23} \\
c_{12} c_{23}-c_{2} s_{12} s_{23} & c_{23} & 1
\end{array}\right)
$$

Elements of $\boldsymbol{g}_{0}$ are obtained as:

$$
\begin{aligned}
& g_{011}=g_{022}=g_{033}=0, g_{012}=g_{021}=-a_{12} s_{12} \\
& g_{013}=g_{031}=\left(d_{2} s_{2} s_{12}-\left(a_{23}+a_{12} c_{2}\right) c_{12}\right) s_{23}-\left(a_{12}+a_{23} c_{2}\right) c_{23} s_{12} \\
& g_{023}=g_{032}=-a_{23} s_{23}
\end{aligned}
$$

The generalised characteristic equation (see equation (19)) is written in terms of the architecture parameters and configuration $\boldsymbol{\theta}$ as:

$$
\begin{aligned}
& 4 s_{2}^{2} s_{12}^{2} s_{23}^{2} h^{3}-4 s_{2} s_{12} s_{23}\left(c_{2} d_{2} s_{12} s_{23}+s_{2}\left(a_{23} c_{23} s_{12}+a_{12} c_{12} s_{23}\right)\right) h^{2} \\
& +\left(\left(-a_{12}^{2}-2 a_{23} c_{2} s_{23}^{2} a_{12}+\left(a_{12}^{2}-a_{23}^{2} c_{2}^{2}\right) c_{23}^{2}-d_{2}^{2} s_{2}^{2} s_{23}^{2}+2 a_{23} c_{2} c_{23} d_{2} s_{2} s_{23}\right) s_{12}^{2}\right. \\
& \left.+2 a_{12} c_{2} c_{12} s_{23}\left(d_{2} s_{2} s_{23}-a_{23} c_{2} c_{23}\right) s_{12}+\left(\left(a_{23}^{2}-a_{12}^{2} c_{2}^{2}\right) c_{12}^{2}-a_{23}^{2}\right) s_{23}^{2}+\sin \left(2 \alpha_{12}\right) \sin \left(2 \alpha_{23}\right) a_{12} a_{23}\right) h \\
& +a_{12} a_{23} s_{12} s_{23}\left(\left(a_{12}+a_{23} c_{2}\right) c_{23} s_{12}+\left(\left(a_{23}+a_{12} c_{2}\right) c_{12}-d_{2} s_{2} s_{12}\right) s_{23}\right)=0
\end{aligned}
$$

As expected, this is a cubic equation, which can be solved in closed-form using Cardano's formula (see, e.g., [17]). For the sake of illustration, we choose the architecture variables as $d_{1}=2, a_{12}=1, \alpha_{12}=\pi / 2, d_{2}=1 / 2, a_{23}=1, \alpha_{23}=\pi / 4, d_{3}=1 / 4, a_{34}=1 / 4$, and the configuration variables as $\theta_{1}=\pi / 6, \theta_{2}=\pi / 4, \theta_{3}=\pi / 2$. For these numerical values, the symbolically computed principal pitches yield the following numerical values:

$$
h_{1}^{h}=-0.987, \quad h_{2}^{h}=0.316, \quad h_{3}^{h}=2.171
$$

The principal screws at this configuration, are given by:

$$
\begin{aligned}
& \hat{\$}_{1}^{h}=(0.745,-0.451,0.493)^{T}+\epsilon(0.940,2.681,-0.972)^{T} \\
& \hat{\$}_{2}^{h}=(0.428,-0.243,-0.870)^{T}+\epsilon(0.510,1.565,-0.550)^{T} \\
& \hat{\$}_{3}^{h}=(0.512,0.859,0.012)^{T}+\epsilon(-1.638,3.503,0.052)^{T}
\end{aligned}
$$

It may be verified that the three principal screws intersect orthogonally at the point:

$$
\boldsymbol{O}^{h}(-0.308,-0.467,-3.207)^{T}
$$




\subsection{A general three system (adopted from [6])}

In this section, we re-work an example provided in [6]. The authors have followed the geometric construction of the pitch-hyperboloid closely. They have solved several linear equations to get to the principal screws, and have thereby obtained the solutions numerically. In our reproduction of the same example we use symbolic computation to obtain the exact solutions. We compare some of the key results to show how our method provides an improvement.

The original screws are specified in terms of their axes, $\boldsymbol{q}_{i}$, a point on the axis, $\boldsymbol{r}_{i}$, and the pitch, $h_{i}, i=1,2,3$. The data are reproduced below:

$$
\begin{aligned}
& \boldsymbol{q}_{1}=(1,0,0)^{T}, \boldsymbol{q}_{2}=(\sqrt{2} / 2, \sqrt{2} / 2,0)^{T}, \boldsymbol{q}_{3}=(0,0,10)^{T} \\
& \boldsymbol{r}_{1}=(0,0,0)^{T}, \boldsymbol{r}_{2}=(0,0,10)^{T}, \boldsymbol{r}_{3}=(0,1,0)^{T} \\
& h_{1}=1, h_{2}=1, h_{3}=-1
\end{aligned}
$$

From these inputs, the $i$ th screw is constructed as $\hat{\boldsymbol{\$}}_{i}=\boldsymbol{q}_{i}+\epsilon\left(h_{i} \boldsymbol{q}_{i}+\boldsymbol{r}_{i} \times \boldsymbol{q}_{i}\right)$. From these screws, the matrices $\boldsymbol{g}, \boldsymbol{g}_{0}$ are computed as:

$$
\boldsymbol{g}=\left(\begin{array}{ccc}
1 & \frac{1}{\sqrt{2}} & 0 \\
\frac{1}{\sqrt{2}} & 1 & 0 \\
0 & 0 & 1
\end{array}\right), \quad \boldsymbol{g}_{0}=\left(\begin{array}{ccc}
2 & -4 \sqrt{2} & 1 \\
-4 \sqrt{2} & 2 & \frac{1}{\sqrt{2}} \\
1 & \frac{1}{\sqrt{2}} & -2
\end{array}\right)
$$

The generalised characteristic equation (19) takes the following form in this case:

$$
4 h^{3}-44 h^{2}-105 h-45=0
$$

Solving this equation, we get the principal pitches as:

$$
\begin{aligned}
& h_{1}^{h}=-3 / 2=-1.500000000 \\
& h_{2}^{h}=(25-\sqrt{745}) / 4 \approx-0.573672032 \\
& h_{3}^{h}=(25+\sqrt{745}) / 4 \approx 13.073672032
\end{aligned}
$$

For the sake of comparison, we quote the corresponding values from [6]:

$$
h_{1}^{h}=-1.562292808, h_{2}^{h}=0.008922166, h_{3}^{h}=13.41848255
$$

The inaccuracy in the result reported in [6] can be attributed to: (a) error accrual due to numerous numerical operations, and (b) computation of the principal pitches from several intermediate (and inaccurate) quantities in the last step. 
Proceeding further, we obtain the principal screws in closed form:

$$
\begin{aligned}
& \hat{\$}_{1}^{h}=\left(\frac{-5}{3 \sqrt{6}}, \frac{-\sqrt{\frac{2}{3}}}{3}, \frac{5}{3 \sqrt{6}}\right)^{T}+\epsilon\left(\frac{10 \sqrt{\frac{2}{3}}}{3}, \frac{-11 \sqrt{\frac{2}{3}}}{3}, \frac{-5}{3 \sqrt{6}}\right)^{T} \\
& \hat{\$}_{2}=\left(-\left(\sqrt{\frac{2}{21605-791 \sqrt{745}}}(-29+\sqrt{745})\right), \frac{5(-27+\sqrt{745})}{\sqrt{43210-1582 \sqrt{745}}}, 2 \sqrt{\frac{2}{21605-791 \sqrt{745}}}\right)^{T} \\
& +\epsilon\left(\frac{706 \sqrt{2}-26 \sqrt{1490}}{\sqrt{21605-791 \sqrt{745}}}, \frac{55(-27+\sqrt{745})}{\sqrt{43210-1582 \sqrt{745}}},-2 \sqrt{\frac{2}{21605-791 \sqrt{745}}}\right)^{T} \\
& \hat{\$}_{3}^{h}=\left((29+\sqrt{745}) \sqrt{\frac{2}{21605+791 \sqrt{745}}}, \frac{-5(27+\sqrt{745})}{\sqrt{43210+1582 \sqrt{745}}}, 2 \sqrt{\frac{2}{21605+791 \sqrt{745}}}\right)^{T} \\
& +\epsilon\left(2(353+13 \sqrt{745}) \sqrt{\frac{2}{21605+791 \sqrt{745}}}, \frac{-55(27+\sqrt{745})}{\sqrt{43210+1582 \sqrt{745}}},-2 \sqrt{\frac{2}{21605+791 \sqrt{745}}}\right)^{T}
\end{aligned}
$$

All the properties of the principal screws (e.g., their pitches, orthogonal alignment, concurrence) can be verified from the above expressions. For instance, the point of concurrence of these screws, i.e., origin of the principal system is obtained as:

$$
\boldsymbol{O}^{h}(0,1 / 2,5)^{T}
$$

For comparison, in [6], the origin is reported as:

$$
\boldsymbol{O}^{h}(1.639061,0.771626,5.80187)^{T}
$$

This example demonstrates very clearly the advantages of the eigenproblem approach to screw theory. In particular, the results obtained are in closed form and therefore exact.

\subsection{A general four system}

In this section we illustrate the procedure described in section 4 by deriving the $h$-basis of an arbitrary general four system. The input four-screw system consists of the following elements:

$$
\begin{aligned}
& \hat{\$}_{1}=(-0.815,0.575,-0.073)^{T}-\epsilon(0.815,-0.575,0.073)^{T} \\
& \hat{\$}_{2}=(-0.799,0.330,0.502)^{T}+\epsilon(-0.799,0.330,0.502)^{T} \\
& \hat{\$}_{3}=(-0.982,0.142,-0.122)^{T}-\epsilon(0.982,-0.142,0.122)^{T} \\
& \hat{\$}_{4}=(-0.992,-0.002,0.129)^{T}-\epsilon(0.992,0.002,-0.129)^{T}
\end{aligned}
$$


The screw system is decomposed in finite, and infinite pitch components, and from infinitepitch screw $\hat{\boldsymbol{\$}}_{T}$, the translation direction is obtained as: $\boldsymbol{z}=(0.488,-0.810,-0.325)^{T}$. The three-system of screws with finite pitches are now decomposed in two sets, with axis parallel and perpendicular to this direction. We choose the free parameter $c_{3}$ in equation (36) as $c_{3}=1 / 3$. The two solutions for the interpolation coefficients $c_{i}$ in equation (34) come out as:

$$
\begin{aligned}
& c_{1}=-0.182, c_{2}=0.925, c_{3}=1 / 3 \\
& c_{1}=0.050, c_{2}=-0.942, c_{3}=1 / 3
\end{aligned}
$$

Correspondingly, the two screws perpendicular to $\boldsymbol{z}$ are, respectively:

$$
\begin{aligned}
& \hat{\$}_{C 1}=-(0.435,0.104,0.393)^{T}-\epsilon(1.729,0.759,-0.501)^{T} \\
& \hat{\$}_{C 2}=(0.119,-0.118,0.474)^{T}+\epsilon(1.870,-0.440,0.375)^{T}
\end{aligned}
$$

The principal screws of the two-system generated by these two screws are:

$$
\begin{aligned}
& \hat{\$}_{1}^{h}=(0.692,0.586,-0.421)^{T}-\epsilon(1.238,-3.067,2.266)^{T} \\
& \hat{\$}_{2}^{h}=(0.532,-0.020,0.847)^{T}+\epsilon(3.512,0.279,-0.100)^{T}
\end{aligned}
$$

The principal screws intersect at the point $\boldsymbol{p}(-1.016,-2.980,-2.210)^{T}$, and their pitches are given by $h_{1}^{h}=1.893, h_{2}^{h}=1.777$ respectively. The principal system of screws can be completed by adding to the pair $\hat{\$}_{1}^{h}, \hat{\$}_{2}^{h}$ two co-axial screws along the $\boldsymbol{z}$ axis, whose pitches are given by $h_{\gamma}$ and $-h_{\gamma}$ respectively, where $h_{\gamma}$ can take any value from $-\infty$ to $\infty$.

We also derive the reciprocal two-system using our procedure. Solving equations (38) in section 4 , the origin of the reciprocal system is computed as:

$$
\boldsymbol{O}^{h}(0.871,-2.972,-2.012)^{T}
$$

The principal screws of the reciprocal system are computed from equation (37) as:

$$
\begin{aligned}
& \hat{\$}_{r 1}=(0.693,0.586,-0.421)^{T}-\epsilon(3.741,-0.651,0.751)^{T} \\
& \hat{\$}_{r 2}=(0.532,-0.020,0.847)^{T}+\epsilon(1.611,0.368,-3.102)^{T}
\end{aligned}
$$

For the purpose of numerical verification, we also compute the reciprocal two-system following the procedure described in [4], and subsequently compute its principal screws using 
our method. The results are in numerical agreement up to the sense of these screws. Further, we generate a twist in the original four-system by random interpolation of the input screws: $\hat{\mathcal{V}}_{o}=(-1.327,0.680,0.005)^{T}+\epsilon(-2.760,-3.152,3.905)^{T}$. It may be verified that the reciprocal products, $\left\langle\hat{\mathcal{V}}_{o}, \hat{\Phi}_{r 1}\right\rangle_{r},\left\langle\hat{\mathcal{V}}_{o}, \hat{\$}_{r 1}\right\rangle_{r} \sim \mathcal{O}(-16)$.

\subsection{The 3-RPS parallel manipulator}

We now analyse a three-degrees-of-freedom fully parallel spatial manipulator to illustrate our method. This manipulator was first proposed by Lee and Shah [10] as a "parallel wrist". Figure 5.4 shows a schematic of this device. The manipulator has three identical prismatic

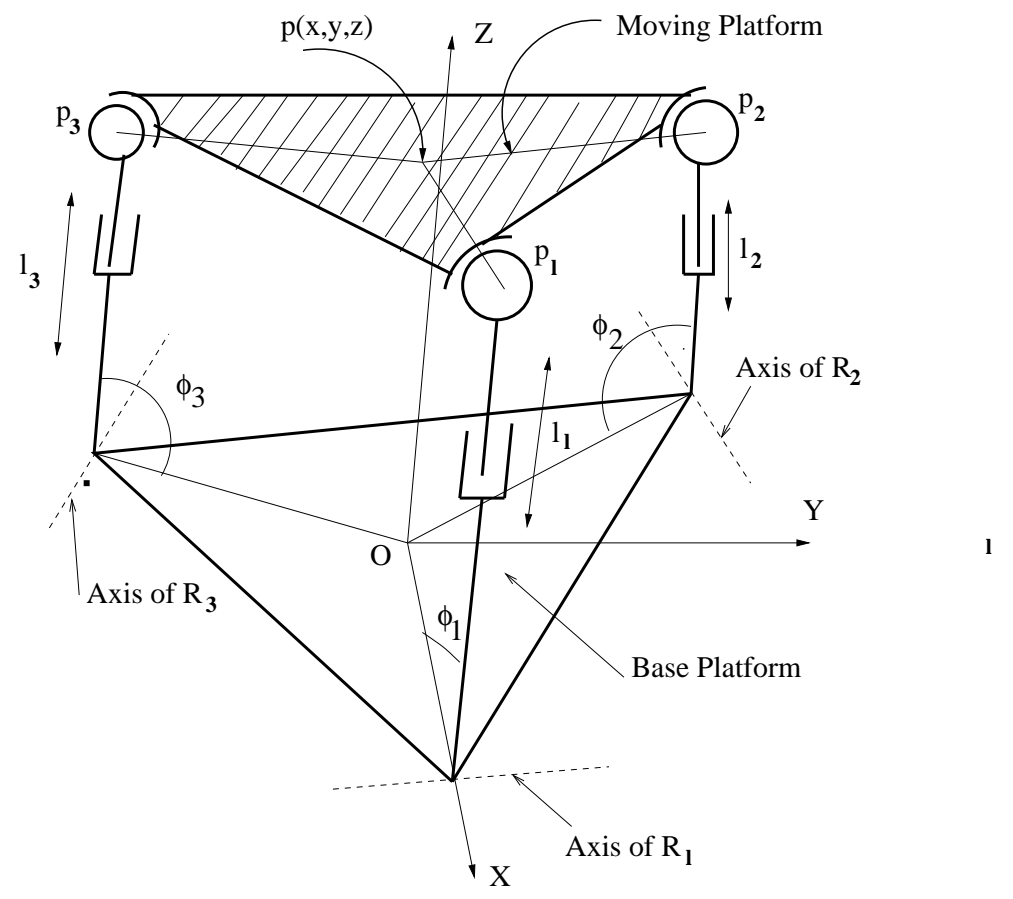

Figure 1: 3-RPS parallel manipulator

legs, each connected to a base with a rotary joint, and to a top platform with a spherical joint. The prismatic joints are actuated, and all other joints are passive. The connection points are distributed uniformly over a circle in each case. The circumradius of the bottom platform (denoted by $b$ ), is taken to be one unit without any loss of generality, and all other linear dimensions are scaled accordingly. The top circumradius is denoted by $a$.

To find the principal screws associated with the motion of the manipulator at any given configuration we must first solve the forward kinematics problem to obtain all the passive variables and then calculate the matrices $\boldsymbol{J}_{\boldsymbol{\omega}}$ and $\boldsymbol{J}_{\boldsymbol{v}}$. We follow the general method presented 
in [12] for calculating these matrices and we retain the same notations.

We take $a=1 / 2$ and choose the configuration given by $l_{1}=3 / 2, l_{2}=5 / 4$, and $l_{3}=3 / 4$. One of the feasible set of solutions for the passive variables is given by $\phi_{1}=0.3947, \phi_{2}=$ 0.9134 , and $\phi_{3}=0.3489$. In this configuration, we have:

$$
\boldsymbol{J}_{\boldsymbol{\eta} \boldsymbol{\phi}}=\left(\begin{array}{lll}
-0.2970 & 1.7070 & 0 \\
0 & 1.8790 & -0.8215 \\
1.7670 & 0 & -0.1425
\end{array}\right), \quad \boldsymbol{J}_{\boldsymbol{\eta} \boldsymbol{\theta}}=\left(\begin{array}{lll}
-1.6710 & -0.0112 & 0 \\
0 & 0.6916 & -1.2780 \\
-1.1620 & 0 & -1.3520
\end{array}\right)
$$

We find that $\operatorname{det}\left(\boldsymbol{J}_{\boldsymbol{\eta} \phi}\right)=-2.3980$, therefore the configuration is non-singular. The matrices $\boldsymbol{J}_{\boldsymbol{\omega}}$ and $\boldsymbol{J}_{\boldsymbol{v}}$ are calculated as:

$$
\boldsymbol{J}_{\boldsymbol{\omega}}=\left(\begin{array}{ccc}
-2.1090 & 0.4495 & 1.3320 \\
-0.3943 & 0.8390 & -1.4990 \\
-0.1334 & 0.4464 & -0.8838
\end{array}\right), \quad \boldsymbol{J}_{\boldsymbol{v}}=\left(\begin{array}{ccc}
-0.4380 & 0.0746 & 0.3199 \\
0.1314 & -0.2365 & 0.3998 \\
1.4230 & 0.5096 & 0.1446
\end{array}\right)
$$

Therefore we get the real and dual parts of $\hat{\boldsymbol{g}}$ as, respectively:

$$
\boldsymbol{g}=\left(\begin{array}{lll}
4.6225 & -1.3385 & -2.0998 \\
-1.3385 & 1.1053 & -1.0539 \\
-2.0998 & -1.0539 & 4.8022
\end{array}\right), \quad \boldsymbol{g}_{0}=\left(\begin{array}{lll}
1.3647 & 0.4166 & -2.8898 \\
0.4166 & 0.1253 & 0.5473 \\
-2.8898 & 0.5473 & -0.6023
\end{array}\right)
$$

The generalised characteristic equation is given by:

$$
4.2633 \times 10^{-14} h^{3}-1.4211 \times 10^{-14} h^{2}+13.3766 h-2.7712=0
$$

It can be seen that the above equation is singular, as the coefficients of $h^{3}, h^{2}$ are practically zeros. The only finite root is obtained as $h_{2}^{h}=0.2072$. It can be shown that the other two roots $h_{1}^{h}, h_{3}^{h}$ tend to $\infty$ while maintaining the relationship $h_{1}^{h}=-h_{3}^{h}$. This confirms the result presented in [9] that the screw-systems associated with certain configurations of the 3-RPS can belong to the seventh special screw-system described by Hunt [4].

As explained in [12], the reason for the degeneracy of the screw-system is that the axes of the rotary joints at the base of the three legs are all in the base plane. This ensures that the legs, and therefore the top platform, cannot have any rotational motion about the normal to the base platform. Therefore at the most two of the angular velocities of the top platform can be linearly independent at any configuration. 
It is intuitive that if any one or two of the rotary joints are twisted out of the base plane then the above degeneracy will be removed. It would result in three independent angular velocities, and the resulting screw system will be a regular one. To confirm this, we repeat our calculations after tilting the axis of the rotary joint of the first leg CCW by an angle $\beta=\pi / 15$ radians about the $\boldsymbol{X}$ axis. The important intermediate entities are found to be as below:

$$
\boldsymbol{J}_{\boldsymbol{\eta} \boldsymbol{\phi}}=\left(\begin{array}{lll}
0.1202 & 1.7960 & 0 \\
0 & 1.8620 & -0.9915 \\
1.9330 & 0 & -0.4282
\end{array}\right), \quad \boldsymbol{J}_{\boldsymbol{\eta} \boldsymbol{\theta}}=\left(\begin{array}{lll}
-1.5340 & -0.2713 & 0 \\
0 & 0.7485 & -1.0720 \\
-1.0990 & 0 & -1.0650
\end{array}\right)
$$

We find that $\operatorname{det}\left(\boldsymbol{J}_{\boldsymbol{\eta} \phi}\right)=-3.5381$, therefore this configuration is also non-singular. The matrices $\boldsymbol{J}_{\boldsymbol{\omega}}$ and $\boldsymbol{J}_{\boldsymbol{v}}$ are calculated as:

$$
\boldsymbol{J}_{\boldsymbol{\omega}}=\left(\begin{array}{lll}
-1.1960 & 0.4041 & 1.2950 \\
-0.7722 & 0.6184 & -1.1520 \\
-0.8876 & 0.3143 & -0.7857
\end{array}\right), \quad \boldsymbol{J}_{\boldsymbol{v}}=\left(\begin{array}{lll}
-0.2945 & 0.1021 & 0.2592 \\
-0.0006 & -0.2295 & 0.2965 \\
1.1050 & 0.6340 & -0.0642
\end{array}\right)
$$

Therefore we get the real and dual parts of $\hat{\boldsymbol{g}}$ as, respectively:

$$
\boldsymbol{g}=\left(\begin{array}{lll}
2.8150 & -1.2400 & 0.0375 \\
-1.2400 & 0.6444 & -0.4358 \\
0.0375 & -0.4358 & 3.6210
\end{array}\right), \quad \boldsymbol{g}_{0}=\left(\begin{array}{ccc}
-1.2560 & -0.2798 & -1.7310 \\
-0.2798 & 0.1972 & 0.1664 \\
-1.7310 & 0.1664 & 0.0893
\end{array}\right)
$$

The generalised characteristic equation is given by:

$$
-4.063 h^{3}-18.2200 h^{2}+6.2830 h-0.4238=0
$$

The three principal pitches are all finite in this case:

$$
h_{1}^{h}=-4.8102, \quad h_{2}^{h}=0.0931, \quad h_{3}^{h}=0.2328
$$

The principal screws of the $h$-basis are computed as:

$$
\begin{aligned}
& \hat{\$}_{1}^{h}=(0.0162,0.3955,-0.9183)^{T}+\epsilon(-0.0186,-0.8045,4.8910)^{T} \\
& \hat{\$}_{2}^{h}=(0.9656,0.2322,0.1170)^{T}+\epsilon(0.2191,-0.0920,0.3643)^{T} \\
& \hat{\$}_{3}^{h}=(-0.2595,0.8886,0.3782)^{T}+\epsilon(-0.0418,-0.3678,1.0820)^{T}
\end{aligned}
$$


It may be verified that these screws meet orthogonally at the point $\boldsymbol{O}^{h}(-1.1957,0.0616,0.0064)^{T}$. These results clearly show that the screw system associated with the 3-RPS manipulator with one out-of-plane leg-joint is a general three-system as per Hunt's classification.

The above example shows how our formulation can be put to use for reliable, and systematic analysis of the screw systems associated with practical manipulators.

\section{Conclusions}

In this paper, we have presented an exact formulation for the derivation of the principal screws of a system of screws. We have shown that the complete solution of the dual eigenproblem in equation (4) leads to a generalised eigenproblem involving the real and dual parts of $\hat{\boldsymbol{g}}$. The eigenproblem is also shown to be solvable in closed form, and particular cases of screw systems have been studied symbolically.

The principal basis of screw systems, derived entirely from a new criterion, namely the extremisation of the pitch of the screws, is shown equivalent to the principal screws discussed in $[3,4]$. The classical results of screw theory have been derived to demonstrate the consistency of our approach. However, the formulation presented here is novel, and some of the closed-form results presented have been derived for the first time. Closed-form and numerical examples have been provided to illustrate the theory developed in this paper as applied to different screw-systems, including those associated with spatial serial and parallel manipulators.

\section{References}

[1] G. Mozzi, Discorso Matematico Sopra il Rotamento Momentaneo dei Corpi (Mathematical treatise on the temporally revolving of bodies). Napoli: Stamperia di Donato Campo, 1763.

[2] M. Chasles, Note sur les Proprietes Generales du Systeme de Deux Corps Semblebles entr'eux. Baron de Ferussac, Paris: Bulletin de Sciences Mathematiques, Astronomiques Physiques et Chimiques, 1830. 
[3] R. S. Ball, A Treatise on the Theory of Screws. Cambridge: Cambridge University Press, 1900.

[4] K. H. Hunt, Kinematic Geometry of Mechanisms. Oxford: Clarendon Press, 1978.

[5] C. G. Gibson and K. H. Hunt, "Geometry of screw systems-2: Classification of screw systems," Mechanism and Machine Theory, vol. 25, no. 1, pp. 11-27, 1990.

[6] W. X. Zhang and Z. C. Xu, "Algebraic construction of the three-system of screws," Mechanism and Machine Theory, vol. 33, pp. 925-930, October 1998.

[7] Y. Fang and Z. Huang, "Analytical identification of the principal screws of the third order screw system," Mechanism and Machine Theory, vol. 33, pp. 987-992, October 1998.

[8] Z. Huang and J. Wang, "Identification of principal screws of 3-DOF parallel manipulator by quadratic degeneration," Mechanism and Machine Theory, vol. 36, pp. 896-911, 2001.

[9] Z. Huang, J. Wang, and Y. F. Fang, "Analysis of instantaneous motions of deficient-rank 3-RPS parallel manipulators," Mechanism and Machine Theory, vol. 37, pp. 229-240, 2002 .

[10] K. M. Lee and D. Shah, "Kinematic analysis of a three-degrees-of-freedom in-parallel actuated manipulator," IEEE Journal of Robotics and Automation, vol. 4, no. 3, pp. 354360, 1988.

[11] Z. Huang, S. H. Li, and R. G. Zuo, "Feasible instantaneous motions and kinematic characteristics of a special 3-DOF 3-UPU parallel manipulator," Mechanism and Machine Theory, vol. 39, pp. 957-970, 2004.

[12] S. Bandyopadhyay and A. Ghosal, "Analytical determination of principal twists in serial, parallel and hybrid manipulators using dual number algebra," Mechanism and Machine Theory, vol. 39, pp. 1289-1305, December 2004.

[13] L. Brand, Vector and Tensor Analysis. New York: John Wiley \& Sons, 1947. 
[14] G. R. Veldkamp, "On the use of dual numbers, vectors, and matrices in instantaneous kinematics," Mechanism and Machine Theory, vol. 11, no. 2, pp. 141-156, 1976.

[15] D. E. Littlewood, A University Algebra. London: The English Language Book Society, 1961.

[16] S. Bandyopadhyay and A. Ghosal, "Geometric characterization and parametric representation of the singularity manifold of a 6-6 Stewart platform manipulator," Mechanism and Machine Theory, vol. 41, pp. 1377-1400, Nov. 2006.

[17] I. N. Herstein, Topics in Algebra. New York: John Wiley \& Sons, 1975.

\section{Appendix}

\section{A Proof of $h^{h}$ being the extreme value of $h$}

From the definitions of $\boldsymbol{g}, \boldsymbol{g}_{0}$, we have:

$$
\begin{aligned}
& \dot{\boldsymbol{\theta}}^{T} \boldsymbol{g}_{0} \dot{\boldsymbol{\theta}}=\dot{\boldsymbol{\theta}}^{T}\left(\boldsymbol{J}_{\boldsymbol{\omega}}^{T} \boldsymbol{J}_{\boldsymbol{v}}+\boldsymbol{J}_{\boldsymbol{v}}^{T} \boldsymbol{J}_{\boldsymbol{\omega}}\right) \dot{\boldsymbol{\theta}}=\boldsymbol{\omega} \cdot \boldsymbol{v}+\boldsymbol{v} \cdot \boldsymbol{\omega} \\
& \dot{\boldsymbol{\theta}}^{T} \boldsymbol{g} \dot{\boldsymbol{\theta}}=\dot{\boldsymbol{\theta}}^{T}\left(\boldsymbol{J}_{\boldsymbol{\omega}}^{T} \boldsymbol{J}_{\boldsymbol{\omega}}\right) \dot{\boldsymbol{\theta}}=\boldsymbol{\omega} \cdot \boldsymbol{\omega}
\end{aligned}
$$

Therefore, from the definition of pitch, we have: $h=\frac{\boldsymbol{\omega} \cdot \boldsymbol{v}}{\boldsymbol{\omega} \cdot \boldsymbol{\omega}}$, i.e, $h=\frac{1}{2} \frac{\dot{\boldsymbol{\theta}}^{T} \boldsymbol{g}_{0} \dot{\boldsymbol{\theta}}}{\boldsymbol{\theta}^{T} \boldsymbol{g} \dot{\boldsymbol{\theta}}}$. To obtain the extreme values of the pitch, we set $\frac{\partial h}{\partial \dot{\boldsymbol{\theta}}}=0$. After a little manipulation, this leads to the condition:

$$
\left(\boldsymbol{g}_{0}-2 h \boldsymbol{g}\right) \dot{\boldsymbol{\theta}}=\mathbf{0}, \operatorname{det}(\boldsymbol{g}) \neq 0
$$

equation (40) is identical with the eigenproblem in equation (9), and it is therefore established that the solution of the generalised eigenproblem gives rise to the extreme values of pitch. 